\title{
Management practices of emergency departments in general hospitals based on blockage of chain of infection during a COVID-19 epidemic
}

\author{
Xiaojing $\mathrm{Hu}^{1} \cdot \mathrm{Si} \mathrm{Liu}^{1} \cdot \mathrm{Bo} \mathrm{Wang}^{2} \cdot$ Hui Xiong ${ }^{2} \cdot$ Ping Wang $^{1}$ \\ Received: 6 July 2020 / Accepted: 5 September 2020 / Published online: 18 September 2020 \\ (c) Società Italiana di Medicina Interna (SIMI) 2020
}

\begin{abstract}
In a Coronavirus disease 2019 (COVID-19) epidemic, management of the emergency department is a difficult task in terms of prevention and control of the disease in general hospitals. On top of meeting urgent needs of patients for medical treatment, the emergency department also has to devote resources into investigation and prevention of COVID-19. At the beginning of the epidemic, with the strategy to intercept the chain of infection, Peking University First Hospital (PKUFH) focused on three important aspects: controlling the source of infection, cutting off the route of transmission, and protecting vulnerable populations, to expeditiously draft scientific and proper management measures for the emergency department, followed by real-time dynamic adjustments based on the development trend of the epidemic. These measures effectively ensured a smooth, orderly and safe operation of the emergency department. As of the writing of this manuscript, there has been no active COVID-19 infection in patients and medical staff in the emergency department, and no infection in patients admitted to PKUFH through the emergency department. This study describes the prevention and control measures in the emergency department of PKUFH during the outbreak of COVID-19, aiming to provide some reference for domestic and international medical institutions.
\end{abstract}

Keywords Coronavirus disease · Coronavirus disease 2019 • Chain of infection · Prevention and control measures · Emergency department management $\cdot$ General hospitals

Hui Xiong

13911875206@163.com

Ping Wang

pingwang40@sina.com

Xiaojing $\mathrm{Hu}$

xiaojing.hu@pkufh.com; hxj_hktk2006@163.com

Si Liu

DocLeo@vip.sina.com

Bo Wang

bjwbdoctor@163.com

1 Medical Affairs Department, Peking University First Hospital, Beijing, China

2 Emergency Department, Peking University First Hospital, Beijing, China

\section{Introduction}

Since December 2019, cases of Coronavirus Disease 2019 (COVID-19) infections had been emerging in Wuhan, Hubei and many other parts of China. Currently, COVID19 is spreading globally. Researchers on the China Novel Coronavirus Investigation and Research Team first discovered and verified that this reported pneumonia was caused by a novel coronavirus (2019-novel coronavirus, 2019nCoV) [1]. The disease mainly manifests as fever, dry cough, and fatigue. Severe patients have dyspnea and may rapidly progress to acute respiratory distress syndrome, septic shock, multiple organ failure, and even death [2, $3]$. The balance between meeting the needs of emergency patients during the epidemic as well as promptly screening and safely transferring high-risk infections to avoid cross-infection among doctors, patients, and medical staff has become an important scientific issue which posed great challenges for emergency departments (ED). It can be said that if the ED is safeguarded, the hospital would 
be in safety. On January 22, 2020, the National Health Commission (herein after referred to as "NHC") of the People's Republic of China issued the Guidelines for the Prevention and Control of New Coronavirus Infection in Medical Institutions (first edition) (National Health Office Medical Letter [2020] No. 65), listing the ED and Fever Clinics as key prevention and control units [4].

A thorough understanding of the chain of infection is crucial for the prevention and control of any infectious disease, as breaking a link anywhere along the chain would stop the transmission of the infectious agent [5], and this theory also holds true for COVID-19. Based on this, PKUFH drafted and formulated a COVID-19 prevention and control strategy to block the chain of infection, and actively practised and explored the three links, controlling the source of infection, cutting off the transmission route, and protecting susceptible populations. This study describes the detailed methods to carry out prevention and control tasks in the ED of PKUFH (Fig. 1).

\section{Methods}

\section{Control of sources of infection}

On March 3, 2020, NHC issued the New Coronavirus Pneumonia Diagnosis and Treatment Program (Trial 7th Edition) (National Health Office Medical Letter [2020]184), pointing out that patients with COVID-19 are the main source of infection [2]. According to the document New Coronavirus Pneumonia Prevention and Control Program (6th Edition) (CDC Letter [2020]204) [6] issued by NHC on March 7, 2020, PKUFH took the following measures to achieve "early detection, early reporting, early isolation, early treatment" and to control the spread of the epidemic to the greatest extent (Table 1).

\section{Establishment of a three-level pre-examination and diagnosis system to promptly detect suspected cases}

(1) Set-up of three 24-h temperature monitoring stations

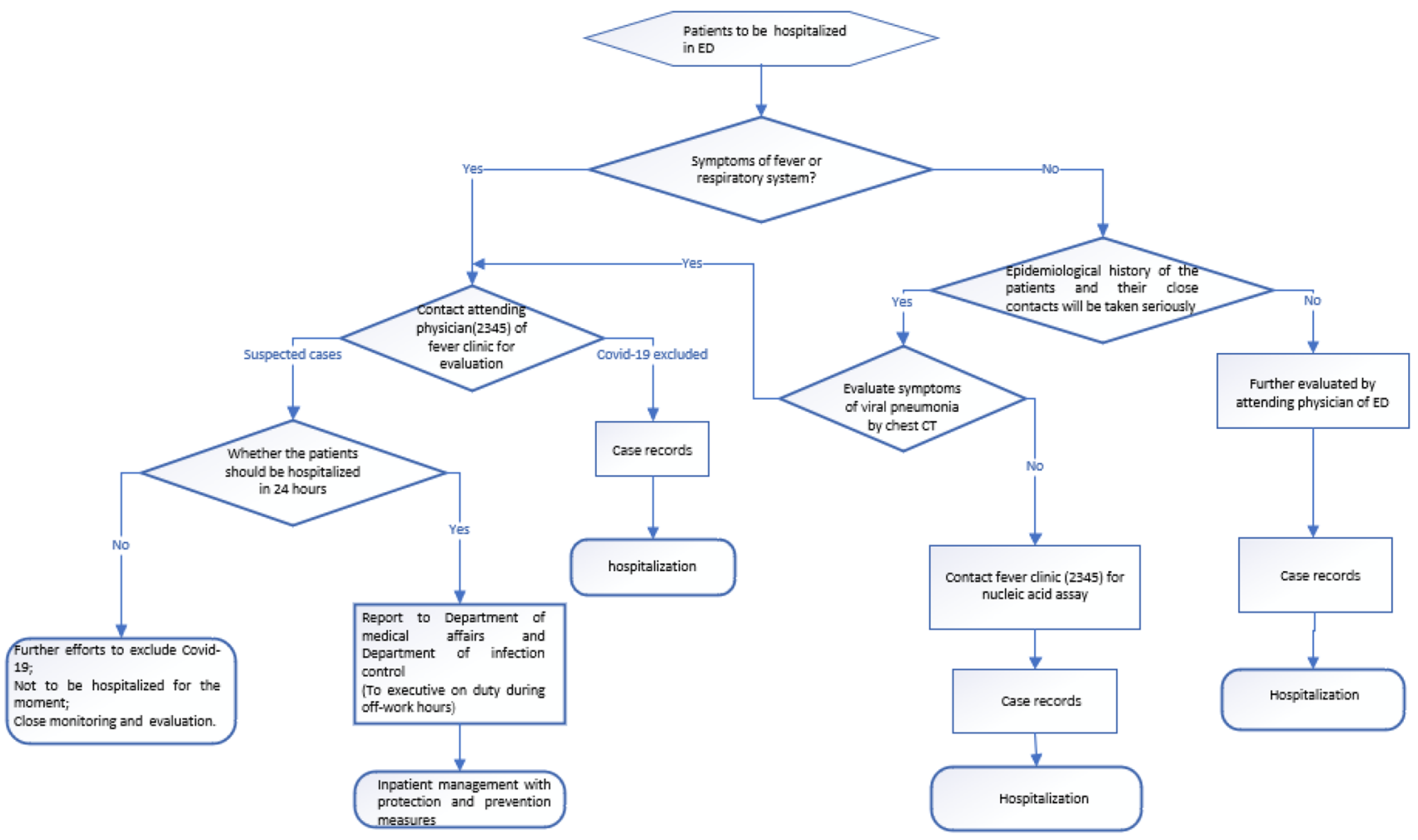

\footnotetext{
*Epidemiological history.

1) travel history or residence history of Wuhan city and surrounding areas, or other communities with case reports within 14 days before the onset:

2) history of contact with 2019-nCov infection (positive nucleic acid test) within 14 days before the onset;

3) history of contact with patients with fever or respiratory symptoms from Wuhan and surrounding areas or from communities with case reports within 14 days

before the onset of the disease;

4) cluster onset
}

Fig. 1 The compliance of emergency treatment standards and admission procedures 
Table 1 Comparison of ED admissions in 2019 and 2020

\begin{tabular}{lllll}
\hline Year lassification & 2019 & 2020 & $\mathrm{Z}$ & P-value \\
\hline Grade 1 & $75(0.3 \%)$ & $125(1.0 \%)$ & - & - \\
Grade 2 & $422(1.7 \%)$ & $338(2.7 \%)$ & - & - \\
Grade 3 & $3,114(12.9 \%)$ & $2073,(16.3 \%)$ & - & - \\
Grade 4 & $20,576(85.1 \%)$ & $10,198(80.1 \%)$ & - & - \\
Total & $24,187(100.0 \%)$ & $12,734(100.0 \%)$ & -12.546 & $<0.01$ \\
\hline
\end{tabular}

24-h temperature monitoring stations were set up at the entrances of the ED, the entrance of the emergency hall, and the emergency triage station. Non-contact infrared temperature monitoring equipment was used at the first two stations, and electronic thermometers with higher accuracy were used at the last station. The responsibilities of the medical staff at these stations were to check patients for body temperatures and proper wearing of masks, and to educate patients on cough etiquette. Patients with fevers (forehead temperature over $37.3^{\circ} \mathrm{C}$ ) were provided with free surgical masks and educated to wear masks properly, and then guided by a designated person to the Fever Clinic following established routes. After ruling out the possibility of COVID-19 infection and recording the patient encounter at the Fever Clinic, patients then returned to the ED. Monitoring for abnormal body temperatures was reported daily to the administrative department for dynamic monitoring.

(2) Conducting epidemiological surveys on all patients and their families

Questionnaire-based survey: the epidemiological questionnaire, based on New Coronavirus Pneumonia Diagnosis and Treatment Program, was designed and updated dynamically. All patients entering the ED were requested to fill out online or paper-based questionnaires at the entrance of the emergency hall. When abnormalities were detected in the online questionnaires, SMS alerts would be sent automatically to the staff. Paper-based questionnaires were to be completed by dyslexia patients with the assistance of on-site staff. Nurses at the emergency triage station were responsible for reviewing questionnaires and providing supplemental information to attending physicians if any abnormities were noticed. After patients entered the clinic, the attending physician would again inquire history of contact and record epidemiological history in the patient's medical record routinely. Nurses at the emergency triage station would summarize abnormal situations and report to the administrative department for dynamic monitoring.

Itinerary verification: personal itinerary inquiry services could be obtained through the epidemic prevention and control communications big data itinerary card, launched by the Information and Communications Administration Bureau of the Ministry of Industry and Information Technology. This service allowed identification of where patients and their families had travelled within the country or abroad in the past 14 days, including foreign countries (regions) and domestic cities (staying over $4 \mathrm{~h}$ ). It was used to determine whether the patient had risks of exposure to an epidemic area.

\section{Timely reporting of suspected cases}

Upon identification of a new suspected case in the ED, fever experts would convene for a group consultation. If the suspected patient was confirmed, the form of "Basic Information Form of COVID-19 suspected patient" was to be filled out, including but not limited to the patient's name, sex, date of birth, ID number, contact phone number, incidence, isolation location, and epidemiological history. Meanwhile, this suspected case would also be reported to the Prevention and Control of Healthcare-associated Infections Department of PKUFH which is responsible for reporting it to higher-level disease control bodies.

\section{Isolation of suspected patients and local treatment}

The suspected patient identified by the ED would be isolated on the spot and given proper medical treatments. The second-line consultation in the Fever Clinic consultation would be conducted if necessary. Once the patient was diagnosed with COVID-19, the ED would report to the Medical Affairs Department which coordinates the transfer of the patient to a designated hospital. People in close contact with the patient would receive medical isolation, and the isolation area would undergo terminal disinfection.

\section{Cutting off transmission routes}

Given that the main transmission routes of the 2019-nCoV are through respiratory droplets and direct contact, virus transmission was minimized to the greatest extent by setting up physical partitions, emergency zoning, reduction in emergency personnel density, and strict cleaning and disinfection.

\section{Set-up of physical partitions, management of travel routes of patients and medical staff}

The temperature of the patient must be measured before entering the ED. Patients with fevers would be guided 
directly to the Fever Clinic located outside of the ED to avoid contact with the medical staff and patients in the ED.

Fences were erected outside of the ED to block access from the ED to the outpatient building, inpatient departments, administrative office areas, to restrict the cross-flow of emergency medical staff and patients to other areas. The two original entrances and exits in the ED were reduced to just one during the epidemic.

Behavioral isolation is equally or even more important than the physical partitioning [7]. The staff in the ED, outpatient building and inpatient departments in PKUFH had fixed and separate positions during the epidemic without overlap.

\section{Reasonable zoning in the ED, use of reserve emergency space, and reduction in personnel density}

According to the requirements of prevention and control of healthcare-associated infections (HAIs), the layout of the ED was rearranged and divided into contaminated area, potentially contaminated area, and clean area. Each area was equipped with single rooms for isolation. Infusion chairs and hospital beds were separated, thereby reducing the personnel density. In addition, the emergency conference room was renovated (with treatment belts, etc.) as an expanded area in anticipation of a surge in patients under observation.

Meanwhile, the number of patients admitted in the established space was reduced. To meet the medical needs of patients, PKUFH immediately added more hospital beds in the emergency hall, a place originally designed for public health emergencies. Screens were set up around each bed as a physical partition to hinder the spread of droplets and for protecting privacy of the patients.

\section{Strengthening maintenance of order and guiding patients to providers in an orderly manner}

Security officers were positioned at the entrance of the ED for order maintenance. According to the principle of "one escort for each patient ", the escort ID was issued to the escort personnel. Patients with self-care ability were not issued with escort cards. More medical guides and medical care providers were assigned to the ED to assist patients with mobility difficulties to obtain medicines and receive examinations.

\section{Reduction in gathering and management of social distancing}

The ED was equipped with self-service machines with functions of registration and payment. As a result, the number of people registering and paying at the manual windows, as well as the waiting time, were reduced.
To remind the patients and escorts to maintain a distance of at least one meter or two seats apart while waiting, signs were posted in waiting areas, such as at the triage stations, cashier desks, pharmacies, and blood collection windows. The signs read "seat should be kept unoccupied" or "empty seat" were posted to the back of the seats accordingly.

\section{Strict compliance of emergency treatment standards and admission procedures, reasonable diversion of patients}

Patients without urgent indications were guided to the Outpatient Departments, and in principle, outpatient patients were not allowed to go to the ED for auxiliary examination.

The ED and the Inpatient Departments worked together to define the COVID-19 rapid detection and admission processes for key critical patients (acute coronary syndrome, acute pancreatitis, gastrointestinal bleeding, etc.). Patients who tested negative for COVID-19 and fell under the admission indications list were to be admitted to the Inpatient Departments timely. The admission process for emergency patients is indicated as follows:

\section{Others}

Professionals of Prevention and Control of Healthcareassociated Infections Department Department visited the ED daily to conduct on-site supervision and improvement, including environment and equipment disinfection, ventilation (such as timely removal of the curtain after a rise in temperature), hand hygiene of medical personnel, and sewage and sewage treatment.

Under the guidance of Prevention and Control of Healthcare-associated Infections Department Department, the General Affairs Department carried out environmental cleaning and disinfection, disposal of dirt and sewage, and ensured ample supply of disinfectants. In addition, they provided food delivery services for medical staff in the ED to ensure nutrition intake and avoid gatherings of people in the cafeteria.

Meetings for information exchange were held online through WeChat Enterprise during the epidemic. Free online consultation services were set up, allowing patients discharged from the ED to receive medical consultation remotely when they had any questions.

\section{Protecting high-risk groups}

2019- $\mathrm{nCoV}$ is a new pathogen to which the population is generally susceptible [8]. The elderly and those with underlying diseases are at high risk. Furthermore, medical staff, who are in close contact with patients and perform high-risk operations, such as sputum aspiration and tracheal intubation, are also COVID-19 high-risk populations. 
Implementation of in-hospital training to increase the attention of medical professionals

The emergency medical staff, temperature monitoring staff, and other personnel (such as medical guidance, nursing workers, security guards, cleaning personnel, etc.) were trained in batches both online and offline. In the training sessions, the relevant knowledge of COVID-19 was introduced to help everyone achieve a reasonable concept of protection. Psychological intervention for medical personnel was also a key point in the training session.

\section{Paying attentions to the health status of medical staff}

Medical professionals returning to Beijing from other provinces must be self-isolated at home. In principle, medical professionals over 60 years old or with fevers and cough would not be allowed to perform medical tasks. The other of medical professionals were assigned to reasonable shift scheduling that guaranteed sufficient rest.

\section{Mutual assistance between departments}

The hospital allocated medical professionals from other departments to help medical professionals in the ED to relieve their workload. Medical personnel assigned to the ED would no longer be allowed to return to the wards or outpatient departments during the epidemic period.

In case of a major rescue effort in the Fever Clinic, the ED should be the back-up rescue force at any time.

\section{Ensuring supply of prevention and control materials for medical professionals}

The ED was equipped with ample medical protective masks, work caps, disposable protection gowns, goggles, latex gloves and other protective equipment.

\section{Statistical analyses}

The numbers and classifications of emergency patients in the ED were collected from January 22 to March 31, 2020, and from the same period in 2019. According to the Guiding Principles for the Piloting of the Classification of Emergency Patients (Draft for comments) issued by the Medical Management Department of the Ministry of Health in 2011 (Medical Note [2011] No. 148), the disease classification was divided into 1-4 grades, defined as grade 1 (endangered patients), grade 2 (critically ill patients), grade 3 (emergency patients) and grade 4 (non-emergency patients).
The percentage was used to describe the disease grades of patients admitted in the ED from January 22 to March 31, 2020 , and from the same period in 2019. Wilcoxon RankSum test was used to compare the changes in different disease grades between the two time periods. Statistical analyses were performed in SPSS 20.0, with $P<0.05$ considered statistically significant.

\section{Results}

\section{Multiple measures to ensure effective prevention from nosocomial infections}

Thirty-three doctors and 116 nurses were included in the ED at PKUFH. From January 22 to March 31, a total of 12,734 patients were admitted to the ED at PKUFH, including 284 patients with fevers who were sent to the fever clinic for treatment. As of 24:00 on March 31, 2020, no COVID-19 infections were reported in the medical professionals and patients in the ED, as well as in the patients admitted to the Inpatient Departments from the ED at PKUFH.

\section{Normal and orderly operations in the ED}

Between January 22 and March 31, 2020, 12,734 patients were admitted to the ED. By contrast, 24,187 patients were admitted during the same period in 2019. The number of emergency patients admitted to the ED in 2020 decreased by $47.4 \%$, compared to the number in 2019 . However, the number of patients with Grade 1 increased by $66.7 \%$ year on year. Significant differences were observed in the composition of the grades of patients admitted to the ED between the two time periods. The summative percentage of Grade 1-3 patients out of the total patients admitted in 2020 was $19.9 \%$, significantly higher than $14.9 \%$ in 2019 . Moreover, the percentage of Grade 1 out of the total patients in 2020 was threefold higher than that of 2019.

\section{Discussion}

\section{Implementation of scientific prevention and control strategies is the most critical factor against COVID-19}

As shown in the result 3.2, the percentage of patients with critical conditions admitted to the ED during the epidemic increased significantly in comparison with the percentage in the corresponding non-epidemic period, showing that there were severe challenges in the management of the 
ED. First, patients underwent COVID-19 screening before being admitted to the Inpatient Departments. The processes of making appointments and pending results delayed the patient's admission time into the hospital. Second, to avoid cross-infection, all wards in the Inpatient Departments were changed to single-patient wards, thereby beds available for patients decreased. Third, the capacity of treatment delivery to patients also decreased as a result of reduced availability of medical professionals because (1) many of them were stranded in other "lockdown" provinces and were unable to return to work, and (2) a large number of them were sent to provide medical support to the city of Wuhan. The aforementioned factors led to prolonged length of stay for high-risk patients in the ED during the epidemic, further increasing the risk of nosocomial infections and the pressure of emergency prevention and control. Therefore, timely and effective measures were of great importance to be taken in the comprehensive management of the ED to defend the "safety line" of the hospital during the epidemic.

Although 2019-nCoV is a new, rare, and highly contagious virus, it does not necessarily indicate that healthcare professionals could not draw experience and references from earlier times of crises. For example, SARS, SARS-CoV, a highly contagious coronavirus that inflicted great harm on humanity [9]. MERS, first reported in Saudi Arabia in 2012, was also caused by a novel coronavirus at the time, MERS-CoV.[10]. Ebola is an acute viral hemorrhagic disease endemic to West Africa [11,12]. These aforementioned viruses were new viruses completely novel to humanity when they were first discovered. However, through infection prevention and control (IPC), a scientific approach and practical solution were designed to prevent harm caused by infection to patients and health workers, the harm caused by SARS, Middle East Respiratory Syndrome, and Ebola virus to humans has been greatly reduced [13]. Such strategies, which have been tested several times based on infectious diseases, demonstrated the key role of IPC. Blocking the chain of infection, an important part of IPC, is constituted of Disease Microorganisms (Agent), Reservoir, Mode of Escape, Mode of Transmission, Mode of Entry, and Susceptible Host. It will stop the transmission of the infectious agent by breaking a link anywhere along the chain [5]. As the emergency department of a large comprehensive hospital, the ED team can choose to block the chain of infection on three aspects: source of infection, transmission routes, and susceptible populations. Our study also proved that for the emergency department, the strategy of blocking the chain of infection (including controlling the source of infection, cutting off the transmission route, and protecting susceptible populations) could effectively prevent and control COVID19 , and maintain normal operation of the emergency department as well as the safety of patients and medical staff during the epidemic to a maximum extent.

\section{The emergency department should not have to fight alone, and cross-department collaboration is needed}

Faced with a deceitful, capricious, and fierce opponent like COVID-19, we cannot simply watch the medical staff in the emergency department fight alone at the frontline, while the others in the hospital make no contributions. If the war on COVID-19 is to be won, forces across multiple departments must be united in the hospital. Studies related to Ebola prevention and control show that a scientific and effective emergency plan is critical for the early response and comprehensive interventions of HAI outbreaks [14]. Therefore, at the very beginning, PKUFH set up the "leading group for prevention and treatment of COVID-19" and "expert group for prevention and diagnosis of COVID-19" by clarifying their respective responsibilities, and formulated a feasible "Working Plan for Diagnosis, Treatment and Prevention of COVID-19". The leading group was responsible for the overall management of the prevention and control tasks, formulation and dynamic adjustment of the prevention and control programs and measures, collection of information and feedbacks, and deployment of medical staff and supplies. The leading group consisted of experts from the head of the hospital, the Prevention and Control of Healthcare-associated Infections Department, the Medical Affairs Department, the Nursing Department, the Pharmacy Department, the Medical Equipment Department, the Human Resources Department, and the General Affairs Department. On the other side, the expert group, consisted of experts from the Infectious Diseases Department, the Emergency Department, the Respiratory Department, the Intensive Medicine Department, the Obstetrics and Gynecology Department, the Pharmacy Department, the Laboratory Department, the Medical Imaging Department, and the infection prevention and control, was responsible for the diagnosis and treatment of COVID-19 and the work of infection prevention and control guidance and training.

\section{Protecting medical staff is equally important as protecting patients}

In COVID-19, as medical staff, it is our undeniable responsibility and obligation to save patients, but this does not mean that medical staff need to make huge and unnecessary sacrifices. As Donatella Lippi once said, we had a great understanding of what infections were and the skills and technology to overcome them. However, doctors, and all healthcare professionals (reflecting the multidisciplinary nature of disease management) still took risks when dealing with pandemics [15]. Also, the emergency medical professionals who were at the anti-epidemic frontline were confronting multi-faceted challenges from the unknown 
nature of the disease, highly-intensive line of work, family, external public opinion, patients and their families [16]. Under such special circumstances, monetary compensation is not of top priority, but rather the available of proven and effective protective gears and equipment. The government and hospitals have the responsibility to protect medical staff. Based on the resolution recommended by the COVID-19 expert group, the ED should be supplied with highest-level personal protective equipment. Medical staff in the ED need to be provided with a sufficient supply of medical protective masks, working caps, disposable isolation clothing, goggles, latex gloves and other protective equipment. To effectively relieve the psychological stress of emergency medical staff and ensure everyone's physical and mental health, PKUFH actively responded to the request of the NHC to integrate psychological crisis intervention into the overall deployment of epidemic prevention and control to alleviate the psychological stress caused by the epidemic [17]. By providing emotional support and psychological intervention, the psychological stress of emergency medical staff was effectively relieved.

\section{Perfecting training and communication mechanism is indispensable}

Studies by Yu IT and others have shown that knowledge, professional skills, and attitude of hospital staff were found to be important in the prevention of HAIs in outbreaks of SARS, Ebola, and MERS in Asia and West Africa [18]. To minimize infection risks of emergency medical staff, temperature monitoring staff and other personnel (such as medical guides, nursing aids, security guards, and cleaning personnel), the Joint Training Group, consisted of experts from the Prevention and Control of Healthcare-associated Infections Department and the Medical Affairs Department, conducted training and assessments. Given the characteristics of different jobs, the group used on-site demonstration teaching, online video, audio teaching, and other methods for training sessions regarding COVID-19 diagnosis and treatments, hand hygiene, use of protective equipment, medical waste disposal, throat swab specimen collection, transfer of suspected patients, and work norms. After the training, all participants were assessed and would only become qualified for deployment after successfully passing the assessments. Field practices have shown that rigorous training and assessments effectively enhance the awareness of medical staff in the ED and other related personnel in protection, and alleviate the panic of all staff to ensure a smooth and orderly work flow in the ED.

In addition, poor and inadequate communication was also found to be a major cause of disease outbreaks in West Africa and Asia [10]. To allow key information flowing efficiently at PKUFH, regular meetings of hospital affairs were held on a daily basis. Every morning, the leadership team of the hospital and main functional departments held a meeting, during which the team summarized work in the previous day, including errors and near misses. The meeting also summarized learnings, questions and challenges, and conducted multi-discipline assessment on issues and PlanDo-Check-Act (PDCA) cycles for test changes. Important information and process changes were shared timely. A Daily Medical Bulletin on the Prevention and Control of COVID -19 was published daily in electronic version. The medical data were released daily, including the number of patient encounters and examinations in the Fever Clinic, number of patient encounters in the ED, number of outpatient encounters, number of patients admitted to each ward, and bed utilization rate. For example, by making the number of beds available in each ward public knowledge across the hospital, and considering patients stranded every day, PKUFH succeeded in transferring emergency patients to the Inpatient Departments for treatment in a timely fashion, thereby effectively alleviating the workload of the ED.

\section{Conclusion}

Based on the three key elements of infectious disease prevention and control, the establishment of scientific and reasonable COVID-19 emergency management measures can effectively ensure the orderly and safe operations of the ED during the epidemic. This paper was drafted in hopes of providing some potential referential value to other healthcare professionals.

\section{Compliance with ethical standards}

Conflict of interest All authors have no potential conflict of interest.

Statements on human and animal rights All procedures performed in this study involving human participants were inaccordance with the ethical standards of the Ethics Committee of Peking University First Hospital research committee and with the 1964 Helsinki declaration and its later amendments or comparable ethical standards.

Informed consent Informed consent was obtained from all individual participants included in this study.

\section{References}

1. Zhu N, Zhang D, Wang W, Li X, Yang B, Song J, Zhao X, Huang B, Shi W, Lu R, Niu P, Zhan F, Ma X, Wang D, Xu W, Wu G, Gao GF, Tan W, China Novel Coronavirus Investigating, and Research Team (2019) A novel coronavirus from patients with pneumonia in China. N Engl J Med 382:727-733 
2. Office of National Health Commission of the people's Republic of China, Office of National Administration of Traditional Chinese Medicine (2020) Notice on the issuance of a new coronavirus pneumonia diagnosis and treatment programme (trial version 7).https://www.nhc.gov.cn/yzygj/s7653p/202003/46c9294a7d fe4cef80dc7f5912eb1989.shtml. Accessed 10 Apr 2020.

3. World Health Organization (2020) The First Few X (FFX) Cases and contact investigation protocol for 2019-novel coronavirus (2019-nCoV) infection. https://www.who.int/publications-detai 1/the-first-few-x-(ffx)-cases-and-contact-investigation-protocolfor-2019-novel-coronavirus-(2019-ncov)-infection. Accessed 10 Apr 2020.

4. Office of National Health Commission of the people's Republic of China (2020) Technical guidelines for the prevention and control of new coronavirus infections in medical institutions (first edition) (medical letter of the National Health Office [2020]65). https:// www.nhc.gov. cn/yzygj/s7659/202001/b91fdab7c304431eb082d67847d27e14.shtml. Accessed 10 Apr 2020.

5. Maguire J, van Seventer NS, Hochberg, (2017) Principles of infectious diseases: transmission, diagnosis, prevention, and control. International encyclopedia of public health, vol 6, 2nd edn. Elsevier, Boston, pp 22-39

6. Office of National Health Commission of the people's Republic of China (2020) Prevention and control of new coronavirus pneumonia (sixth edition).https://www.nhc.gov.cn/jkj/s3577/20200 3/4856d5b0458141fa9f376853224d41d7.shtml. Accessed 10 Apr 2020.

7. Liuyi L, An'hua W (2020) Confusion on prevention and control of healthcare-associated infection of novel coronavirus. Chin J Infect Control 19: 105-108

8. Anhua $\mathrm{Wu}$, Xun H, Chunhui Li, Liuyi Li (2020) Novel coronavirus (2019-nCoV) pneumonia in medical institutions: problems in prevention and control. Chin J Infect Control 19:99-104

9. Ge XY, Li JL, Yang XL, Chmura AA, Zhu G, Epstein JH et al (2013) Isolation and characterization of a bat SARS-like coronavirus that uses the ACE2 receptor. Nature 503:535-538

10. Cotten M, Watson SJ, Kellam P, Al-Rabeeah AA, Makhdoom HQ, Assiri A et al (2013) Transmission and evolution of the Middle East respiratory syndrome coronavirus in Saudi Arabia: a descriptive genomic study. Lancet 382:1993-2002
11. Ebola is an acute viral hemorrhagic disease endemic to West Africa. Zhao JM, Dong SJ, Li J, Ji JS(2015) The Ebola epidemic is ongoing in West Africa and responses from China are positive. Mil Med Res 2:9

12. Gire SK, Goba A, Andersen KG, Sealfon RS, Park DJ, Kanneh L et al (2014) Genomic surveillance elucidates Ebola virus origin and transmission during the 2014 outbreak. Science 345:1369-1372

13. World Health Organization (2016)Guidelines on core components of infection prevention and control programmes at the national and acute health care facility level. https://www.who.int/gpsc/ipccomponents/en/. Accessed 10 Jun 2020.

14. Modeling the effect of comprehensive interventions on Ebola virus transmission (2015) Sci Rep5:15818.

15. Lippi, D., Bianucci, R. \& Donell, S. Role of doctors in epidemics: historical perspectives and implications for COVID-19(2020) Intern Emerg Med.https://doi.org/10.1007/s11739-020-02351-x

16. World Health Organization (2020) Coronavirus disease (COVID19) outbreak: rights, roles and responsibilities of health workers, including key considerations for occupational safety and health.https://www.who.int/publications/i/item/coronavirus-disea se-(covid-19)-outbreak-rights-roles-and-responsibilities-of-healt h-workers-including-key-considerations-for-occupational-safet y-and-health. Accessed 10 Apr 2020.

17. Department of Disease Prevention and Control of National Health Commission of the people's Republic of China (2020) Notice on Guiding Principles of Emergency Psychological Crisis Intervention for New CoronavirusPneumonia Epidemic. https://www.nhc. gov.cn/jkj/s3577/202001/6adc08b966594253b2b791be5c3b94 67.shtml. Accessed 10 Apr 2020.

18. Yu IT, Li Y, Wong TW, Tam W, Chan AT, Lee JH et al (2004) Evidence of airborne transmission of the severe acute respiratory syndrome virus. N Engl J Med 350:1731-1739

Publisher's Note Springer Nature remains neutral with regard to jurisdictional claims in published maps and institutional affiliations. 